\title{
Research on GPS/INS Integrated Navigation Algorithm
}

\author{
Ming Liu \\ Flying College, Binzhou University, Shandong, China
}

\begin{abstract}
The GPS/INS integrated navigation system has a strong complementarities among the subsystems. It has a very broad application prospects and an important role. In this paper, the development of the satellite navigation system and the inertial navigation system is introduced, and the current situation of integrated navigation system is analyzed. In the meanwhile, this paper briefly introduces the GPS/INS integrated technology. the mathematical model of GPS/INS integrated navigation system is analyzed, and the system mathematical model that takes position and speed as the viewing measurement is obtained. At last, the algorithm of integrated navigation is analyzed, and the extended Kalman filtering algorithm is proposed and simulation analysis and performance comparison of these algorithms are carried out.
\end{abstract}

Keywords-GPS/INS integrated navigation; system model; kalman filtering

\section{INTRODUCTION}

The so-called navigation system refers to the system that provides information to the control system that transport the carrier that will be carried from the starting point to the destination[1]. Navigation system measures and calculates the instantaneous motion state and location of shipping carrier, to provide to the driver or automatic driving instrument to achieve accurately manipulate or control the carrier.

In the ancient times when science and technology is not developed, there is example that humans have made use of the Big Dipper to navigate, which can be considered as the earliest application of navigation. And in modern times, along with the continuous development of science and technology, navigation information resources that human beings can choose and use become more and more and types of navigation system are more and more various[2, 3]. There are many kinds of navigation system that can be used, such as GPS system, GLONASS system, Doppler navigation system, air data system, OMEGA system, Loran C navigation system, magnetic heading system and inertial navigation system.

All of these navigation systems can combine to form an integrated navigation system. But comparatively speaking, what is widely used all over the world at present and the most advanced in performance is the GPS/INS integrated navigation system[4 6].

\section{INTEGRATED NAVIGATION SYSTEM ANALYSIS}

\section{A. Inertial Navigation System}

The inertial navigation system developed in early 20th century, because it is a kind of autonomous navigation system and has no information exchange with the outside world, its concealment is not good and the work is not restricted by any outside conditions. This unique feature makes it become a widely-used navigation method in the field of aviation, spaceflight and navigation. Since the seventies of the 20th century, inertial sensor and inertial navigation system technology made great development and progress, and there are various types of inertial sensors such as laser gyro, optical fiber gyroscope, pressure electric vibrating gyroscope, electrostatic supported gyro, hemispherical resonator gyro and so on.

There are two schemes in the development of the inertial navigation system. And these two kinds of schemes are classified by different installation methods of inertial navigation system. The inertial measurement instrument is mounted on the table of the carrier of inertial platform, and this scheme is platform inertial navigation system. The inertial measuring instrument is directly mounted on the carrier. This scheme is called the strap-down inertial navigation system[7].

At present, the two schemes of inertial navigation system are coexisting. The two schemes have their own advantages and disadvantages, and according to their respective advantages and disadvantages, two kinds of inertial navigation schemes are different in use. The strap-down inertial navigation system is superior to the platform inertial navigation system in terms of reliability, volume, weight and cost, and the former is better than the latter. But in the aspect of precision, the latter is better than the former. Therefore, in the specific application of these two schemes, it should be based on the actual situation of the carrier to choose the kind of scheme.

\section{B. Global Positioning System}

The first satellite navigation system put into use in the world is the United States navy navigation satellite system, which also becomes a transit system. The system was put into use in 1964, and open for the global civil in 1967. But because of time gap of the system in the cover, it makes users cannot get continuous positioning and each location takes a long time. In addition, as it is not suitable for high dynamic user and positioning accuracy, it spurs that people to pursue for new more ideal satellite navigation system. GPS comes into being just under this background[8].

The GPS is mainly divided into three parts, the space part, the ground control part and the user equipment part. The specific functions of each part are as follows.

The space part mainly refers to GPS satellite navigation 
constellation composed of GPS.

The GPS ground control part is composed of master station and monitoring stations distributed in the whole world, upward station and the communication network that connects them together.

The user equipment part mainly refers to the GPS receiver. The GPS receiver, through observing and capturing the visible satellite, calculates the position and the speed of the receiver by using navigation message sent by satellite to complete the function of navigation and positioning.

\section{GPS / INS Integrated Navigation System}

GPS and INS as the earliest mature research satellite navigation system and the ground navigation system, whose combination is relatively easy in design and implementation. Integrated navigation system avoids the single shortcomings of each other, and then make the comprehensive utilization of the accuracy of the navigation accuracy be better than that of two systems separately working, which are mainly embodied in the following. GPS/INS integrated navigation system has the ability to resist external interference. When the satellite system receiver becomes out of action, or when the GPS signal is subjected to high intensity interference, the INS can still run and use alone[9].

Once the GPS receiver restore the operation state, the GPS receiver can immediately obtain the information in current state from the INS system, which can let the GPS system rapidly reobtain GPS code and carrier in the shortest period of time and achieve the rapid restoration of the GPS system's performance. GPS receiver antenna can also make use of the auxiliary information provided by INS navigation system to help the receiver antenna fast lock GPS satellite signal, and then control the impact of interference factors on the system.

\section{RESEARCH AND APPLICATION OF GPS/INS INTEGRATED NAVIGATION ALGORITHM}

\section{A. Mathematical Model of GPS/INS Integrated Navigation System \\ 1) State equation}

To establish the state equation of the GPS/INS integrated navigation system, it is necessary to determine the form and dimension of each state variable in the state equation. Assume that the system state variables are 8 in number, namely location error in three directions, speed error in three directions, a clock and a clock error drift rate. This paper selects platform misalignment angle, velocity error, position error and gyro and accelerometer bias as the state variables.

$$
X(t)=\left[\phi_{x}, \phi_{y}, \phi_{z}, \delta v_{x}, \delta v_{y}, \delta v_{z}, \delta L, \delta \lambda, \delta h, \varepsilon_{x}, \varepsilon_{y}, \varepsilon_{z}, \nabla_{x}, \nabla_{y}, \nabla_{z}\right]^{T}
$$

where, $\phi_{x}, \phi_{y}$ and $\phi_{z}$ is platform error angle; $\delta v_{x}, \delta v_{y}$ and $\delta v_{z}$ is the velocity error along the east, north and up direction; $\delta L$ and $\delta \lambda$ is latitude, and longitude error. The rest are gyroscopes and accelerometers constant error, and noise modeling of gyroscopes and accelerometers re zero mean white noise.

The state equation is shown in equation (2).

$$
\dot{X}(t)=F(t) X(t)+G(t) W(t)
$$

Wherein $W$ is irrelevant white noise with zero mean, and it satisfied $E\left[W(t) W^{T}(\tau)\right]=Q(t) \delta(t-\tau)$.

$F(t)$ is the state transition matrix of dimensions of 16 multiplied by 16 .

\section{2) Measurement equation}

In this paper, the loose combination is applied, in which the position and velocity of the strap-down inertial navigation system and the GPS receiver are taken as the external viewing measurements. In this paper, we choose the two-dimensional position difference and the two-dimensional velocity difference as the external viewing measurements of the system.

\section{B. Application of Kalman Filtering Algorithm in GPS/INS Data Fusion}

In 1960, Kalman put forward the theory of Kalman filtering theory. At the same time, along with the rapid development of computer technology, especially continuous progress of digital computing technology, the Kalman filtering is applied to various fields and it has obtained successful application, and it becomes the key of research and application in the field of autonomous navigation.

The standard Kalman filtering algorithm is expressed by a series of recursive mathematical formulas. This algorithm can not only effectively estimate the state, but also let the estimation of mean square error be the minimum, which can not only estimate past and current state of the signal, but also can estimate the future status of the signal, even don't know the exact nature of the model.

For general nonlinear system, it is difficult to achieve strict recursive filtering in theory, so most of the algorithms implement linear handling of the system by approximately approach means. Among them, extended Kalman filter algorithm proposed by Sahara and Bucy is such kind of linear method. This algorithm makes use of measuring equation and state equation, measurement error and white noise statistical characteristics in the system to make Taylor series expansion of the nonlinear function in the optimal estimation point, and then abandon high order component, so as to realize the partial linearization in the nonlinear system. At last, through the filtering basic equations, derive linear recursive filtering equation.

The specific steps of the extended Kalman filtering algorithm are as follows.

(1) The initial values: $x_{k-1}, P_{k-1}$; 
(2) Calculate the estimated value: $\left.\hat{x}_{k}\right|_{k-1},\left.\hat{Z}_{k}\right|_{k-1}$;

(3) Calculate the coefficient: $\hat{A}_{k}, \hat{B}_{k}, \hat{H}_{k}, \hat{J}_{k}$;

(4) Calculate the predicting characteristic value: $\left.\hat{P}_{k}\right|_{k-1}$;

(5) Calculate the increasing profit of extended Kalman filtering: $\mathrm{K}_{\mathrm{EKF}}$;

(6) Calculate the estimated value: $\hat{x}_{k}, \hat{P}_{k}$;

(7) Iteration: $x_{k}=\hat{x}_{k}, P_{k}=\hat{P}_{k}$;

(8) Let $\mathrm{k}=\mathrm{k}+1$, and repeat the steps of (2) - (7).

Although the algorithm is the algorithm relatively simple and easy to implement in nonlinear filtering algorithm, the algorithm still has defects. In the implementation process of the algorithm, it is necessary to proceed linear handling in allusion to the system model, and the state equation of the system is approximated by the local linearization of the state of the prediction system. In such an approximation, the linearization error cannot be avoided, especially in some strongly nonlinear systems, the results are often not satisfactory. Moreover, in the implementation process of the EKF algorithm, the matrix Jacobians of the system need to be calculated. The higher the system dimension also directly improves the computational difficulty and fault tolerance of the matrix Jacobians, which also increases the complexity of the EKF algorithm implementation.

\section{SIMULATION AND ANALYSIS}

Because the update rates of strap-down inertial guide data and GPS data are different, in this paper, through the computer, complete the data synchronization. Then, make use of the mathematical model of the GPS/INS integrated navigation system obtained to make simulation results verification and analysis of the extended Kalman filter algorithm.

The initial latitude of the system locates is $L=39^{\circ}$, longitude $\lambda=106^{\circ}$, altitude $h=5500 \mathrm{~m}$, terminal latitude $L=37^{\circ}$, longitude $\lambda=108^{\circ}$, and altitude $h=4000 \mathrm{~m}$. The earth's rotation rate $\omega_{i e}=7.29212 \times 10^{-5} \mathrm{rad} / \mathrm{s}$, acceleration of gravity $g=9.7803267714 \mathrm{~m} / \mathrm{s}^{2}$; random constant drift of gyro is $0.01 \% / h$, random drift $0.01 \% / h$; random constant drift of accelerometer is $100 \mu \mathrm{g}$, random drift for $5 \mu \mathrm{g}$; initial level and the azimuth misalignment angle are 90", 90", and 120"; initial velocity error $\delta v_{x}=\delta v_{y}=0.1 \mathrm{~m} / \mathrm{s}$; initial position error $\delta L=3^{\circ} ; \delta \lambda=3^{\circ}$. Simulation results are as shown in the Figure 1 and Figure 2.

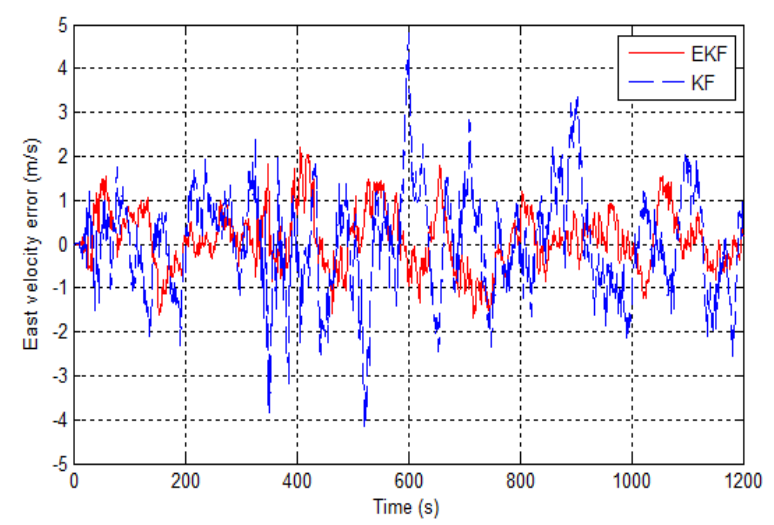

FIGURE I. EAST VELOCITY ERROR

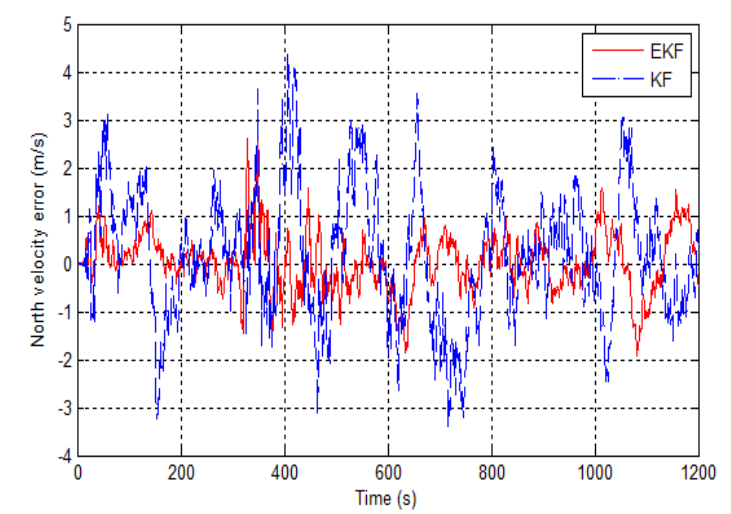

FIGURE II. NORTH VELOCITY ERROR

From the above simulation results in Figure 1 and Figure 2, the simulation calculation results showed that, the results of $\mathrm{KF}$ algorithm and EKF algorithm are the same as that of integrated navigation system data fusion. EKF algorithm has a higher accuracy, because the system is non-linear. So the general EKF algorithm is widely used.

\section{CONCLUSION}

GPS/INS integrated navigation system is the world's most widely used integrated navigation system because of the complementarities among the subsystems. As a main research direction of GPS/INS integrated navigation system, data fusion filtering technology is becoming the focus and key point. In this paper, the integrated navigation data fusion filtering technology is studied. Inertial navigation system and global positioning system is introduced, the typical integrated navigation system data fusion algorithm is analyzed, mainly including the extended Kalman filtering algorithm, and simulation analysis and performance comparison are proceeded on the two typical algorithms.

\section{ACKNOWLEDGMENT}

The authors would like to thank the anonymous reviewers for many constructive comments which lead to a significant improvement of the paper. The authors would also like to thank funding source who has contributed to this research. 
The author was supported by Shandong Provincial Natural Science Foundation, China (ZR2014FL020).

\section{REFERENCES}

[1] Han S, Wang J. Integrated GPS/INS navigation system with dual-rate Kalman Filter[J]. GPS Solutions. 2012, 16(3): 389-404.

[2] Wu Y, Wang J, Hu D. A New Technique for INS GNSS Attitude and Parameter Estimation Using Online Optimization[J]. IEEE Transactions on Signal Processing. 2014, 62(10): 2642-2655.

[3] Daum F. Nonlinear filters: beyond the Kalman filter[J]. IEEE Aerospace and Electronic Systems Magazine. 2005, 20(8): 57-69.

[4] Chang G. On kalman filter for linear system with colored measurement noise[J]. Journal of Geodesy. 2014, 88(12): 1163-1170.

[5] Chang G. Alternative formulation of the Kalman filter for correlated process and observation noise[J]. IET Science, Measurement \& Technology. 2014, 8(5): 310 - 318.

[6] Chang G. M-estimator based robust Kalman filter for systems with process modeling errors and rank deficient measurement models[J]. Nonlinear Dynamics. 2015, accepted.

[7] Chang G. Robust Kalman filtering based on Mahalanobis distance as outlier judging criterion[J]. Journal of Geodesy. 2014, 88(4): 391-401.

[8] Yang Y, Gao W, Zhang X. Robust Kalman filtering with constraints: a case study for integrated navigation[J]. Journal of Geodesy. 2010, 84: 373-381.

[9] Huang G, Zhang Q. Real-time estimation of satellite clock offset using adaptively robust Kalman filter with classified adaptive factors[J]. GPS Solutions. 2012, 16(4): 531-539. 